\title{
MDSC and beyond: a symposium-in-writing on myeloid cells with immunoregulatory activity by members of the Mye-EUNITER network
}

\author{
Sven Brandau ${ }^{1,2,3}$ (D)
}

Published online: 18 March 2019

(c) Springer-Verlag GmbH Germany, part of Springer Nature 2019

In a healthy person, myeloid immune cells provide protection against invading harmful pathogens or malignant cells, whilst maintaining a state of unresponsiveness ('tolerance') to our own body tissues and to harmless substances we eat or inhale. Insufficient activity of myeloid and other immune cells permits pathogens to cause morbidity and mortality and fails to prevent the progression of single malignant cells to cancer. Traditionally, myeloid immune cells have been regarded as anti-microbial and immunostimulatory cells. More recently, additional regulatory and even immunosuppressive functions have been described for the major myeloid cell types, neutrophils, dendritic cells, and macrophages. Furthermore, initial murine studies have described the induction and pathologic function of so-called myeloidderived suppressor cells (MDSC). MDSC are a heterogeneous mixture of immature and mature myeloid cells, which share two major common denominators: (1) pathologic expansion in disease and (2) immunosuppressive activity. After discovery in mice, human counterparts are the focus of intensive contemporary research.

Not unexpectedly for an emerging field, research on this regulatory function of myeloid cells [herein referred to as myeloid regulatory cells (MRC)] and their biology and pathophysiological relevance has developed in a noncoordinated and fragmented fashion. In fact, research has rather evolved in a cell-specific manner, resulting in individual research groups having excellent expertise and technical tools for just one particular subtype of MRC, such as

Sven Brandau

sven.brandau@uk-essen.de

1 Research Division, Department of Otorhinolaryngology, University Hospital Essen, Hufelandstrasse 55, 45122 Essen, Germany

2 West German Cancer Center, Essen, Germany

3 German Cancer Consortium, Partner Site Essen, Essen, Germany
MDSC, neutrophils, macrophages or DC. Furthermore, a comprehensive and comparative analysis of MRC subtypes is missing, especially due to the lack of commonly accepted specific markers, which could be utilized by the scientific community to unequivocally identify these cells. This generates controversial and unclear results even among research groups working on the same cell type. In addition, if compared to $T$ cells, the dysfunction of myeloid cells and the molecular mechanisms leading to their dysfunction, are less well characterized.

In order to facilitate exchange among those "research communities" and facilitate comparability of research, in 2014 the European Network of Investigators Triggering Exploratory Research on Myeloid Regulatory Cells (MyeEUNITER) was formed, funded by the COST programme of the European Union. In the context of this COST Action we are referring to MRC as a heterogeneous group of myeloid cells which acquire immunoregulatory and/or immunosuppressive activity as a consequence of the disease of the host. The term "regulatory" is used with reference to the far better characterized regulatory $\mathrm{T}$ cells, which in contrast to classical $\mathrm{T}$ cells are not immune effector cells, but rather downregulate immune responses. As stated above, similar phenomena have been observed for myeloid cells; examples include but are not limited to immunosuppressive granulocytes, tolerogenic dendritic cells (DC), regulatory macrophages and the heterogeneous group of MDSC.

In a 2018/2019 symposium-in-writing in Cancer Immunology, Immunotherapy members of the Mye-EUNITER network review and discuss distinct and overarching activities of MRC in cancer, infection, tissue homeostasis and tolerance. A comprehensive analysis and discussion of technical aspects of MDSC isolation and functional analysis is also presented. Amodio et al. [1] discuss beneficial functions of MRC as they maintain tissue homeostasis and are currently explored for their potential to reduce over-shooting immunity in inflammatory diseases and autoimmunity. A 
comprehensive overview on distinct and overlapping tumorpromoting regulatory activity of monocytes/macrophages, neutrophils, MDSC, and myeloid DC is provided by Umansky and colleagues [2]. Dorhoi and coworkers [3] focus on the emerging role of MDSC in infections, which appears to depend on pathogen type and molecular mechanisms of virulence. Technical aspects of MDSC isolation and phenotyping in patients, mice and non-human primates are debated by Cassetta and colleagues [4]. A critical and comprehensive appraisal of experimental approaches measuring immunosuppressive activity of MDSC (the defining key function of this cell type) is provided by Bruger and co-authors [5].

Improved comparability of immunomonitoring and cell biological studies together with a broader understanding of the clinical and disease-related relevance of MRC will make these cells promising biomarkers and potential therapeutic targets in the future.

Funding This work was supported by COST (European Cooperation in Science and Technology) and the COST Action BM1404 MyeEUNITER (http://www.mye-euniter.eu). COST is part of the EU Framework Programme Horizon 2020.

\section{Compliance with ethical standards}

Conflict of interest The author declares that he has no conflict of interest.

\section{References}

1. Amodio G, Cichy J, Conde P, Matteoli G, Moreau A, Ochando J, Oral BH, Pekarova M, Ryan EJ, Roth J, Sohrabi Y, Cuturi
MC, Gregori S (2018) Role of myeloid regulatory cells (MRCs) in maintaining tissue homeostasis and promoting tolerance in autoimmunity, inflammatory disease and transplantation. Cancer Immunol Immunother. https://doi.org/10.1007/s0026 2-018-2264-3

2. Umansky V, Adema GJ, Baran J, Brandau S, Van Ginderachter JA, Hu X, Jablonska J, Mojsilovic S, Papadaki HA, Pico de Coaña, Y, Santegoets KCM, Santibanez JF, Serre K, Si Y, Sieminska I, Velegraki M, Fridlender ZG (2018) Interactions among myeloid regulatory cells in cancer. Cancer Immunol Immunother. https:// doi.org/10.1007/s00262-018-2200-6

3. Dorhoi A, Glaría E, Garcia-Tellez T, Nieuwenhuizen NE, Zelinskyy G, Favier B, Singh A, Ehrchen J, Gujer C, Münz C, Saraiva M, Sohrabi Y, Sousa AE, Delputte P, Müller-Trutwin M, Valledor AF (2018) MDSCs in infectious diseases: regulation, roles, and readjustment. Cancer Immunol Immunother. https://doi. org/10.1007/s00262-018-2277-y

4. Cassetta L, Baekkevold ES, Brandau S, Bujko A, Cassatella MA, Dorhoi A, Krieg C, Lin A, Loré K, Marini O, Pollard JW, Roussel M, Scapini P, Umansky V, Adema GJ (2019) Deciphering myeloid-derived suppressor cells: isolation and markers in humans, mice and non-human primates. Cancer Immunol Immunother. https://doi.org/10.1007/s00262-019-02302-2

5. Bruger AM, Dorhoi A, Esendagli G, Barczyk-Kahlert K, van der Bruggen P, Lipoldova M, Perecko T, Santibanez J, Saraiva M, Van Ginderachter JA, Brandau S (2018) How to measure the immunosuppressive activity of MDSC: assays, problems and potential solutions. Cancer Immunol Immunother. https://doi.org/10.1007/ s00262-018-2170-8

Publisher's Note Springer Nature remains neutral with regard to jurisdictional claims in published maps and institutional affiliations. 